\title{
Lithium as Probe of the Scenarios of the chemical enrichment of the Galaxy
}

\author{
P. François \\ ESO and DASGAL (Observatoire de Paris), Casilla 19001, Santiago \\ 19, Chile \\ V. Hill \\ ESO and DASGAL-Observatoire de Paris, Karl Schwarzschild Strasse \\ 2, Garching bei München, D-85748, Germany \\ M. Spite and F. Spite \\ DASGAL and UMR G8633 du CNRS, Observatoire de Paris, F-92195 \\ Meudon CEDEX, France
}

\begin{abstract}
Jehin et al. (1999) find that, in a sample of moderately metal-poor stars, a group is rich in $s$ elements, and they propose an enrichment by accretion of matter by the winds of AGB stars. We tried to check the implications for the lithium abundances.
\end{abstract}

\section{Introduction}

Recently Jehin et al. (1999) presented very careful determinations of abundances in a sample of 21 mildly metal-poor stars, $(-1.3<[\mathrm{Fe} / \mathrm{H}]<-0.8)$, in particular, the abundances of the heavy elements. They show that the abundance of Europium, an element formed by the $r$ process (rapid addition of neutrons) is uniformly correlated with the abundance of the $\alpha$ elements (like magnesium and titanium). This is expected, since both $\alpha$ elements and $r$ elements are supposed to be produced and ejected by the same kind of massive supernovae (SN II).

On the contrary, the behaviour of the $s$ elements (produced by slow addition of neutrons), relative to the $\alpha$ elements is not linear (cf. the behaviour of Yttrium in Fig. 1). In a first group of stars $(0.0<[\mathrm{Ti} / \mathrm{Fe}]<0.23)$ there is a good correlation between $[\mathrm{Y} / \mathrm{Fe}]$ and $[\mathrm{Ti} / \mathrm{Fe}]$, but in the second group $[\mathrm{Ti} / \mathrm{Fe}]$ is gathered in the narrow range $0.23-0.25$, whereas the spread in $[\mathrm{Y} / \mathrm{Fe}]$ is large. This second group is (in average) Y-rich.

\section{A scenario}

Jehin et al. suggest a scenario based on two distinct phases of chemical enrichment: a first phase by the products of supernovae explosions of massive stars (SN II) a second phase by the stellar winds from intermediate mass AGB stars. Generally, the AGB produce both lithium and $s$ process elements. In this case it can be expected that the second group (Y-rich) is also Li-rich. In some cases AGB stars produce $s$ elements without lithium, and then their stellar wind will, on the contrary dilute $\mathrm{Li}$ and enhance $\mathrm{Y}$ in the observed stars. 


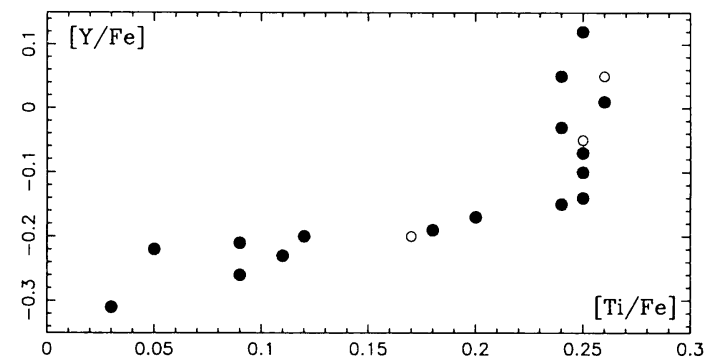

Figure 1. The first group $([\mathrm{Ti} / \mathrm{Fe}]<0.23)$ shows a good correlation between $[\mathrm{Y} / \mathrm{Fe}]$ and $[\mathrm{Ti} / \mathrm{Fe}]$, the second group is $\mathrm{Y}$-rich with a large Y spread. The open circles represent the stars where the lithium line could not been detected

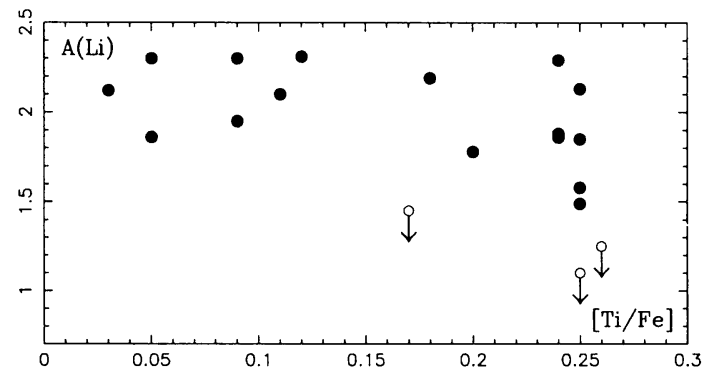

Figure 2. The first group has a rather constant $\mathrm{Li}$ abundance (typical of metal-poor stars), the second group ([Ti/Fe] $>0.23$ ) has a larger spread in lithium and a lower mean abundance

We have measured the lithium abundance for 19 stars (out of the 21 stars). Most of the spectra have been obtained with FEROS at the ESO $1.5 \mathrm{~m}$ telescope and three of them with the CFH telescope in Hawaii. The results are presented in Fig. 2 (similar to Fig. 1 but with the lithium abundance as ordinate). All these stars are dwarfs and their temperature is higher than $5700 \mathrm{~K}$.

The first group $([\mathrm{Ti} / \mathrm{Fe}]<0.23)$ behaves like metal-poor stars : a rather constant $\mathrm{Li}$ abundance, but the second group $([\mathrm{Ti} / \mathrm{Fe}]>0.23)$ has a larger spread and a smaller mean $\mathrm{Li}$ abundance suggesting an anticorrelation $\mathrm{Li} / \mathrm{Y}$ by Li-dilution. But within this last group there is no clear correlation (or anticorrelation) star by star, between $\mathrm{Y}$ and $\mathrm{Li}$. The temperature distributions in the two groups are rather similar, and thus the difference of lithium behaviour cannot be attributed to different temperature distributions. Our data do not bring a clear confirmation of the interpretation proposed by Jehin et al., the problem obviously deserves further investigation.

\section{References}

Jehin E., Magain P., Neuforge C., Noels A., Parmentier G., Thoul A. A. , 1999, A\&A 341, 241 